\title{
Surgical management of acute necrotizing lung infections
}

\author{
Beth Ann Reimel $M D^{1}$, Baiya Krishnadasen $M D^{1}$, Joseph Cuschieri $M D^{1}$, Matthew B Klein $M D^{1}$, \\ Joel Gross $\mathrm{MD}^{2}$, Riyad Karmy-Jones MD ${ }^{1,3}$
}

\begin{abstract}
BA Reimel, B Krishnadasen, J Cuschieri, MB Klein, J Gross, R Karmy-Jones. Surgical management of acute necrotizing lung infections. Can Respir J 2006;13(7):369-373.
\end{abstract}

BACKGROUND: Surgical resection for acute necrotizing lung infections is not widely accepted due to unclear indications and high risk. OBJECTIVE: To review results of resection in the setting of acute necrotizing lung infections.

METHODS: A retrospective review of patients who underwent parenchymal resection between January 1, 2000, and January 1, 2006, for management of necrotizing pneumonia or lung gangrene.

RESULTS: Thirty-five patients underwent resection for lung necrosis. At the time of consultation, all patients presented with pulmonary sepsis, and also had the following: empyema $(n=17)$, hemoptysis $(n=5)$, air leak $(n=7)$, septic shock requiring pressors $(n=8)$ and inability to oxygenate adequately $(n=7)$. Twenty-four patients were ventilated preoperatively. Eleven patients had frank lobar gangrene, and the other patients had combinations of necrotizing pneumonia and abscesses. In 10 patients, preresection procedures were performed, including percutaneous drainage of an abscess $(n=4)$, thoracoscopic decortication $(n=4)$ and open decortication $(n=2)$. Procedures included pneumonectomy $(n=4)$, lobectomy $(n=18)$, segmentectomy $(n=2)$, wedge resection $(n=4)$ and debridement $(n=7)$. There were three $(8.5 \%)$ postoperative deaths - two due to multiple organ failure and one due to anoxic brain injury. All patients not ventilated preoperatively were weaned from ventilatory support within three days. Of those ventilated preoperatively, three died, while four remained chronically ventilator dependent.

CONCLUSIONS: Surgical resection for necrotizing lung infections is a reasonable option in patients with persistent sepsis who are failing medical therapy. Ventilated patients have a worse prognosis but can still be candidates for resection. Patients who are hemodynamically unstable appear to have better outcomes if they can be stabilized before resection.

Key Words: Lung resection; Necrotizing pneumonia

\section{Traitement chirurgical de la pneumonie nécrosante aiguë}

HISTORIQUE : La résection chirurgicale dans les cas de pneumonie nécrosante aiguë n'est pas largement utilisée en raison de ses indications floues et du risque élevé qui y est associé.

OBJECTIF : Passer en revue les résultats de la résection dans le contexte de la pneumonie nécrosante.

MÉTHODES : Revue rétrospective des résections du parenchyme réalisées entre le $1^{\text {er }}$ janvier 2000 et le $1^{\text {er }}$ janvier 2006 pour le traitement de la pneumonie nécrosante, ou gangrène pulmonaire.

RÉSULTATS : Trente-cinq patients ont subi une résection pour nécrose pulmonaire. Au moment de leur consultation, tous les patients présentaient une infection pulmonaire en plus des éléments suivants : empyème ( $\mathrm{n}=17)$, hémoptysie $(\mathrm{n}=5)$, fuite d'air $(\mathrm{n}=7)$, choc septique justifiant le recours aux amines pressives $(\mathrm{n}=8)$ et incapacité de s'oxygéner adéquatement $(\mathrm{n}=7)$. Vingt-quatre patients étaient sous ventilateur avant l'intervention. Onze patients présentaient une gangrène lobaire franche et les autres présentaient à la fois une pneumonie nécrosante et des abcès. Chez 10 patients, une prérésection a été effectuée, notamment par drainage percutané des abcès $(n=4)$, décortication thoracoscopique $(n=4)$ et décortication ouverte $(n=2)$. Les interventions ont entre autre été : pneumonectomie $(n=4)$, lobectomie $(n=18)$, segmentectomie $(n=2)$, résection cunéiforme $(n=4)$ et débridement $(n=7)$. On a dénombré trois décès postopératoires $(8,5 \%)$, deux causés par une défaillance multiviscérale et l'autre, par une lésion ayant conduit à l'anoxie cérébrale. Tous les patients qui n'étaient pas sous ventilateur avant l'intervention ont été sevrés de l'assistance respiratoire en l'espace de trois jours. Parmi les patients qui se trouvaient sous respirateur avant l'intervention, trois sont décédés, tandis que quatre sont demeurés dépendants de l'appareil. CONCLUSIONS : La résection chirurgicale dans la pneumonie nécrosante est une option envisageable chez les patients dont l'infection persiste malgré l'administration d'un traitement pharmacologique. Le pronostic des patients sous respirateur est plus sombre, mais ils demeurent de bons candidats à la résection. Les patients qui sont hémodynamiquement instables semblent mieux s'en sortir s'ils peuvent être stabilisés avant la résection.
In the modern era, necrotizing pneumonia is an uncommon Iillness for which the treatment is generally supportive. Indications for surgical intervention are limited to specific complications, namely, persistent or major hemoptysis, abscess, empyema and lung gangrene (1). Surgical approaches are complicated by a number of factors, including patient stability, extent of lung injury, magnitude of intervention required and whether a residual space is present or anticipated. Unlike soft tissue infections, indications for resection for acute pulmonary necrotizing infections are not well established. Building on our initial experience with lung gangrene, we have developed an aggressive approach for patients who have persistent signs of pulmonary infection and/or associated complications despite adequate medical therapy (1).

\section{METHODS}

A retrospective review of patients who underwent parenchymal resection between January 1, 2000, and January 1, 2006, for necrosis, abscesses or gangrene was performed. All procedures were performed at Harborview Medical Center (Seattle, Washington), a level I trauma centre and county hospital that serves as a tertiary referral base for the regions of Washington, Wyoming, Alaska,

${ }^{1}$ Department of Surgery; ${ }^{2}$ Department of Radiology, Harborview Medical Center, Seattle; ${ }^{3}$ Department of Surgery, Southwest Washington Medical Center, Vancouver, Washington, USA

Correspondence: Dr Riyad Karmy-Jones, Southwest Washington Medical Center, Physicians' Pavilion, 200 NE Mother Joseph Place, Suite 300,

Vancouver, Washington 98664, USA. Telephone 360-514-1854, fax 360-514-6063, e-mail rkarmyjo@swmedicalcenter.com 


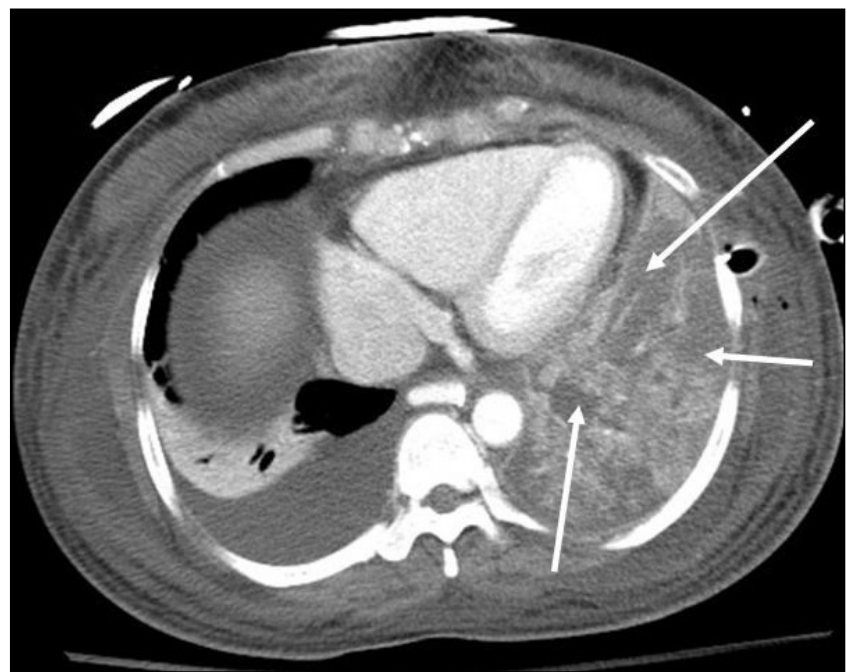

Figure 1) Computed tomography scan in a patient with necrotizing pneumonia. Note the patchy areas without contrast uptake (arrows)

Montana and Idaho. In the present study, more than $80 \%$ of patients had no insurance, and perhaps as many as one-third were indigent. Necrotizing pneumonia was defined as patchy inflammation, with microabscesses and a lack of perfusion on computed tomography (CT); an abscess was defined as a cavitary lesion occupying less than $50 \%$ of the affected lobe; and gangrene was defined as a lack of perfusion with central necrosis affecting more than $50 \%$ of the involved lobe (Figures 1 and 2).

The primary indication for resection was pulmonary sepsis, as defined by fever, leukocytosis and positive sputum cultures in patients with radiographic evidence of parenchymal necrosis. If patients had septic physiology, requiring active fluid or vasopressor resuscitation, resection was deferred until hemodynamic stability was attained. In this setting, attention was directed toward draining any empyema either operatively or by image-directed drainage, and any large parenchymal cavities were similarly drained. CT scans with intravenous contrast were obtained in all cases before resection. Ultimately, the decision to perform resection was based on finding a 'target' area that appeared to be the primary source of ongoing signs of infection, which was, in many cases, associated with prolonged respiratory failure and increased metabolic requirements. All patients had, at minimum, persistent elevations in leukocyte count and fever, despite systemic antibiotic therapy as directed by a positive sputum culture and, if performed, pleural or parenchymal culture results.

When the necrotizing process involved the periphery of the parenchyma, and the more central lung tissue was viable, debridement was performed. If the base of a lower lobe or apex of an upper lobe was involved but the more central portions were viable and were thought to be able to hold staples, generous nonanatomical wedge resections were used. Otherwise, anatomical resections were performed with use of muscle flaps to reinforce bronchial stumps or cover raw parenchyma, depending on the degree of tissue edema in the chest wall at the time of surgery and the stability of the patient. An irrigation system, using a Jackson-Pratt drain, was placed after lobectomy or pneumonectomy if it was thought that the chest wall tissue could be closed sufficiently to avoid leakage through the wound.

\section{RESULTS}

A total of 35 patients underwent resection for necrotizing parenchymal infections during the time period studied. At the time of consultation, all patients presented with pulmonary

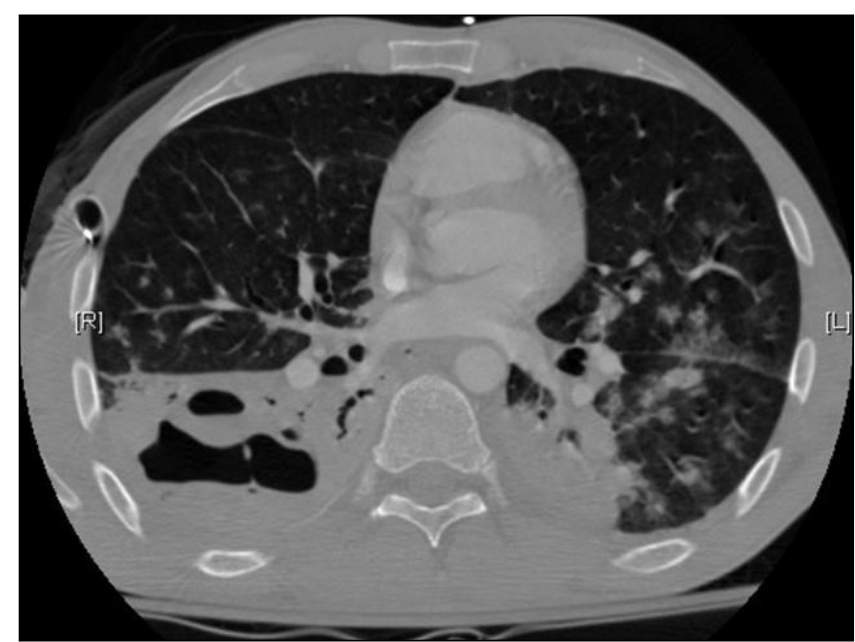

Figure 2) Patient with lung gangrene of the right lower lobe. There is consolidation with patchy perfusion in the right lower lobe surrounding a large cavitary lesion

sepsis, as defined by fever, leukocytosis and positive sputum cultures; patients also presented with the following: empyema $(n=17)$, hemoptysis $(n=5)$, persistent air leak $(n=7)$, septic shock requiring vasopressors $(n=8)$ and inability to oxygenate adequately $(n=7)$. Eleven patients had frank gangrene affecting at least one lobe, and the other patients had necrotizing pneumonia with varying degrees of abscess formation. The time from admission to initial surgical consultation was within two weeks in all but three cases, and surgery was performed within a further two weeks in all but five cases. In 10 patients, including the eight patients who were in septic shock requiring resuscitation, preresection procedures were performed, including percutaneous drainage of abscesses $(n=4)$, thoracoscopic decortication $(n=4)$ and open decortication $(n=2)$. Twenty-four patients were ventilated before resection, of whom 10 required a positive end-expiratory pressure greater than $10 \mathrm{cmH}_{2} \mathrm{O}$ and a fraction of inspired oxygen greater than $80 \%$ to maintain or attempt to maintain oxygenation. In addition, five of these patients were being managed with permissive hypercapnia. Eight of these ventilated patients had diffuse parenchymal inflammation involving all lobes, in addition to the underlying necrotizing changes.

The operations were performed via posterolateral thoracotomy in 32 cases and anterolateral thoracotomy in three cases. A single lumen endotracheal tube was required in 10 cases, a single lumen tube with endobronchial blocker in eight cases and a double lumen tube in the remaining cases. Procedures included pneumonectomy $(n=4)$, lobectomy $(n=18)$, segmentectomy $(n=2)$, wedge resection $(n=4)$ and debridement $(n=7)$. Three of the four pneumonectomies were right-sided. Lobectomy involved the right lower lobe in eight cases, middle lobe in one case, right upper lobe in three cases, left lower lobe in four cases and left upper lobe in two cases. Latissimus muscle flaps were raised in five cases in which debridement was performed, as well as in five cases of lobectomy and three cases of pneumonectomy. For patients in whom debridement was performed, the muscle flap was used to fill the residual space. In the lobectomy and pneumonectomy cases, the flap was used to buttress the bronchial stump.

The most common pathogens cultured from the lung tissue were Streptococcus pneumoniae (15 cases) and Staphylococcus aureus (11 cases). Of the $S$ pneumoniae cases, seven were resistant 
to penicillin, while six of the $S$ aureus cases were resistant to methicillin. In six cases, the primary organism cultured was Pseudomonas aeruginosa and, in the remainder of cases, there were mixed flora, including Klebsiella and Haemophilus species.

Of note, in six lobectomy cases, the initial CT scan had suggested diffuse parenchymal involvement of the entire lung on that side, and in the case of five debridements and one wedge resection, the entire lobe was initially diffusely inflamed. However, over the time before surgery, the areas of the lung that had documented perfusion tended to resolve, further localizing the area that actually required resection, as opposed to areas that were involved with inflammation but were viable.

There were no deaths at surgery or within $24 \mathrm{~h}$; however, three patients $(8.5 \%)$ died postoperatively. One patient died on the second postoperative day due to persistent respiratory failure with concomitant cardiovascular instability. The second patient had marked improvement in respiratory and infectious parameters, but during weaning, it became apparent that he had suffered severe anoxic brain injury during the period before operation and support was withdrawn. The third patient (admitted following burns with inhalation injury) initially improved and was on minimal ventilator support, but then developed progressive wound and new pulmonary sepsis, and died 10 days after resection. These three cases were among the eight with diffuse bilateral parenchymal involvement. All three had undergone lobectomy.

There was one case of postoperative wound infection requiring local wound debridement, which occurred in a patient whose latissimus muscle was mobilized as a flap. Bronchial stump leak occurred after one lobectomy in which a muscle flap had been used to reinforce the stump, and after one in which the latissimus muscle was not mobilized. In the former case, the leak was sealed with simple drainage, and in the latter case, it required open drainage. Including these patients, a total of four patients who underwent lobectomy or pneumonectomy developed postoperative empyema requiring repeat drainage. Three of the cases occurred among the six patients not receiving an irrigation system compared with a single occurrence among the 16 patients who did $(\mathrm{P}=0.02)$.

All patients not ventilated preoperatively survived, and all were weaned from ventilatory support within five days of operation, although one patient subsequently was readmitted for respiratory failure, which ultimately required tracheotomy and long-term ventilator care, following a second episode of alcohol withdrawal and aspiration. Of the patients ventilated preoperatively, four remained chronically ventilator dependent after six months to two years of follow-up; these four patients were those who had been receiving maximal ventilator support, and two of these were among the group who had bilateral diffuse parenchymal inflammation/infection. Thus, among the eight patients who were both ventilated preoperatively and who had radiographic evidence of diffuse bilateral inflammation, three died and two remained chronically ventilator dependent.

Of note, thoracic service was consulted for 17 other patients in whom patchy areas of lung necrosis were documented. None of these patients had a persistent septic course, and they were followed clinically with interval CT scans until discharge. In 10 of these cases, decortication was performed thoracoscopically. In some cases, residual pneumatoceles remained, but the bulk of the parenchyma recovered. In all cases, CT with contrast showed that the majority of the lung parenchyma was perfused, suggesting that medical therapy could be successful.

\section{DISCUSSION}

Necrotizing pneumonia, lung abscesses and lung gangrene represent a spectrum of parenchymal destruction that can be loosely defined by the degree of inflammation, necrosis, time course, degree of sepsis and radiographic patterns, although all three can coexist. Necrotizing pneumonia has been characterized radiographically by findings of consolidated lung with peripheral necrosis and multiple small cavities, and may be rapidly progressive; the predominant clinical feature is often acute respiratory failure. Lung gangrene, in its most classic form, is defined radiographically by central vascular or bronchial obstruction with larger abscess collections, often with obvious necrotic debris floating in them (1). These three entities are separated from classic 'destroyed lung' (including chronic tuberculosis) by the latter's chronic course, which usually presents as an indolent, progressive infection that is similar to bronchiectasis $(2,3)$. In addition, necrotizing pneumonia and its complications are characterized by various degrees of vascular obstruction, which correlate with the risk of failure of medical therapy $(4,5)$.

The predominant organisms associated with complicated necrotizing pneumonia or lung gangrene are Klebsiella pneumoniae, $P$ aeruginosa and $S$ pneumoniae $(1,6)$. Of note, the incidence of streptococcus pneumonia in this setting may be increasing (7-12). Lung gangrene has also been described in other more 'chronic' settings such as tuberculosis $(13,14)$.

The clinical presentation and course of patients with necrotizing pneumonia is highly variable, and depends on the overall health of the patient and the organisms responsible for the infection. All patients with necrotizing pneumonia have fever, cough and putrid breath, and those with more indolent infections have weight loss. Despite clinical findings of sepsis, sputum and blood cultures may be negative in up to $50 \%$ of cases (12). Patients typically have a protracted illness that may be further complicated by empyema, bronchopleural fistulae and/or lifethreatening hemoptysis. It is not uncommon for chest radiography to underestimate the degree of parenchymal destruction noted on CT (15).

The risk of developing complications of necrotizing pneumonia that require surgical intervention is also not well defined. Donnelly and Klosterman (15), in a review of children with complicated pneumonia (defined as those not responding to antibiotics), noted that of 56 chest CT scans, parenchymal complications were present in 40 cases and pleural complications were present in 37 cases. CT scans are clearly superior in evaluating the disease process. Apart from the detection of occult changes, such as microabscesses, CT can detect areas devoid of perfusion, which can be indicative of an increased risk of developing extensive local necrosis as well as failure of medical management. As the infection evolves, the cavities coalesce to form a single or a few larger cavities, which contain sloughed lung tissue that floats on pus collected in the bottom of the abscess. This is responsible for the 'air crescent' sign characteristic of necrotizing pneumonia with pulmonary gangrene (16).

The natural history and management of lung abscesses (in the absence of surrounding parenchymal necrosis) has been better defined than necrotizing pneumonia. Specific indications for medical management, surgical resection and percutaneous drainage have been established (17). This is not the case for necrotizing pneumonia and lung gangrene, which differ from 'simple' abscesses in that they tend to involve more lung 
parenchyma, be less discrete, and have different success rates with percutaneous drainage and antibiotic therapy. For example, percutaneous drainage of necrotizing pneumonia is associated with increased complications. Hoffer et al (18) noted a $100 \%$ failure rate and a $70 \%$ rate of bronchopleural fistula when percutaneous drainage was used to treat liquefying necrotizing pneumonia as opposed to a discrete, well-defined abscess. Nevertheless, in patients with dominant cavitary lesions who have necrotizing pneumonia, this approach may still be effective as a temporizing measure.

Lung gangrene, in particular, is distinguished by the development of a central vascular obstruction, obstruction of the bronchus and, in most cases, significant cavitation. There is not the same firm fibrous capsule that characterizes the 'typical' abscess. Gangrene with cavitary changes is often difficult to distinguish from lung abscesses, but the lack of a firm fibrous capsule or finding a 'mass within the mass' (consistent with necrotic debris in a cavity of pus) can help $(16,19)$. While the air crescent sign has been considered a characteristic finding, it is not uniformly present $(7,16)$. However, the key distinguishing features of bronchial obstruction, or more commonly lack of perfusion, are detected by CT (with intravenous contrast) and predict the failure of medical management $(4,20)$. Moon et al (6) followed 11 patients with Klebsiella pneumonia. Nine patients had evidence of diffuse parenchymal changes with areas of patchy necrosis. Eight developed empyema, and in two cases, small abscess cavities coalesced to form cavitary changes consistent with gangrene. The mortality rate ranges from $17 \%$ with a 'localized' form to $40 \%$ with 'diffuse' changes (21).

The principles of surgical management of established gangrene are a combination of the approaches taken for hemoptysis, empyema and abscesses. In particular, contralateral aspiration during surgery can result in terminal respiratory failure and mandates lung exclusion if there are large central cavitary changes (3). However, the timing of surgery is not clear. There are limited data indicating that, in general, operative resection is ultimately associated with better outcomes than medical therapy alone. Schamaun et al (11) reported on 14 patients with 'massive' unilateral pulmonary gangrene. Four of these patients were treated medically alone, and all four died. The other 10 patients underwent surgical resection (predominantly pneumonectomy), and all survived. Hammond et al (7) commented on two patients with sepsis and destruction of one lung, both of whom underwent emergent pneumonectomy and survived. In general, treating empyema before lung resection to stabilize patients with acute sepsis requiring resuscitation seems advisable (22). In patients who do not respond to therapy, and who are considered prohibitive operative risks, various staged 'fenestration' procedures may be considered to allow for stabilization (23). The negative impact of this approach is that it tends to leave the pleural space densely consolidated, making subsequent resection more difficult. Alternatively, if the majority of cavitation is peripheral, it can be debrided, although this may be associated with an increased risk of late complications, including bleeding and air leak (24). A significant number of patients also have empyema at the time of surgery, and the risk of postoperative pleural space infection and stump leak, particularly if pneumonectomy is required, is markedly increased unless preventive measures are taken (2).

In the present study, 11 patients had defined gangrene, while the remaining patients had necrotizing pneumonia with clear, large abscess formation in some cases, and diffuse, small, multifocal cavitary changes in others. Despite medical therapy, all patients had persistent fever and leukocytosis, and the majority were unable to be weaned from the ventilator. CT identified primary target areas, as indicated by a lack of perfusion to a lobe or frank necrosis/gangrene. The operation can be performed with a single lumen tube, although using a bronchial blocker facilitates exposure and reduces the risk of contralateral aspiration. The key technical issues are to identify the pulmonary artery early, which, in the case of lung gangrene, is surprisingly easy because the parenchymal tissue tends to shred away and the pulmonary artery in the fissure can be found with simple blunt dissection. Necrotizing pneumonia without frank gangrene is more difficult because the parenchyma is heavy and dense, and in many cases, it is easier to ligate the pulmonary vein first; this allows the lung to be retracted with greater ease to identify either the proximal main pulmonary artery or the artery in the fissure proximal to the lower lobe on either side, which then allows dissection into the fissure. In either circumstance, there should be no hesitation to divide more than one rib to gain the exposure needed.

Except in patients with diffuse bilateral parenchymal changes, resection was well tolerated. In a number of patients, resection appeared to resolve the underlying pulmonary infection, which, in turn, permitted weaning. However, resection was not performed in acutely unstable patients. These patients were initially managed by pleural drainage, image-directed drainage and decortication. This appeared to be beneficial by allowing for a more controlled resection, despite the fact that a significant number of patients still required aggressive ventilator support. Peripheral-based lung lesions could be debrided and covered with muscle flaps, which appeared to eliminate the problem of air leak. Irrigation systems appear to reduce the risk of recurrent empyema after lobectomy and pneumonectomy. We still advocate covering the stump, particularly after pneumonectomy, with viable tissue to reduce the risk and severity of postresection bronchopleural fistula.

\section{CONCLUSIONS}

Clearly, we do not have a true 'denominator' in the present study because we do not know how many patients had evidence of necrotizing pneumonia but did not get a surgical consult. However, we do think that we have demonstrated that parenchymal resection, from debridement to pneumonectomy, may be a reasonable option in patients with necrotizing lung infections who are failing medical therapy. CT scans can predict the likelihood of failure of medical therapy, and can be used to follow the course of such infections to determine whether the process is improving, stabilizing or getting worse. Patients may require serial CT scans every $48 \mathrm{~h}$ to $72 \mathrm{~h}$, which, in conjunction with their clinical progress, may indicate the need for surgical intervention. Candidates are patients who still have signs of infection but do not require vasopressors at the time of surgery. Patients with diffuse bilateral parenchymal inflammation and who are ventilator dependent have the worst prognosis and the greatest likelihood of remaining ventilator dependent. Moreover, in this subset, unless there is clear evidence that the infection is not being controlled medically, it may be better to avoid surgery. Nevertheless, ventilated patients can still be appropriate candidates for resection if there is continued evidence that the parenchymal necrotizing process is likely the source of persistent sepsis. 


\section{REFERENCES}

1. Krishnadasan B, Sherbin VL, Vallieres E, Karmy-Jones R. Surgical management of lung gangrene. Can Respir J 2000;7:401-4.

2. Blyth DF. Pneumonectomy for inflammatory lung disease. Eur J Cardiothorac Surg 2000;18:429-34

3. Stevens MS, de Villiers SJ, Stanton JJ, Steyn FJ. Pneumonectomy for severe inflammatory lung disease. Results in 64 consecutive cases. Eur J Cardiothorac Surg 1988;2:82-6.

4. Curry CA, Fishman EK, Buckley JA. Pulmonary gangrene: Radiological and pathologic correlation. South Med J 1998;91:957-60.

5. Gutman E, Rao KV, Park YS. Pulmonary gangrene with vascular occlusion. South Med J 1978;71:772-5.

6. Moon WK, Im JG, Yeon KM, Han MC. Complications of Klebsiella pneumonia: CT evaluation. J Comput Assist Tomogr 1995; 19:176-81.

7. Hammond JM, Lyddell C, Potgieter PD, Odell J. Severe pneumococcal pneumonia complicated by massive pulmonary gangrene. Chest 1993;104:1610-2.

8. Isaacs RD. Necrotizing pneumonia in bacteraemic pneumococcal infection. Br J Dis Chest 1986;80:295-6.

9. Kothari PR, Jiwane A, Kulkarni B. Pulmonary gangrene complicating bacterial pneumonia. Indian Pediatr 2003;40:784-5.

10. Collazos J, Fernandez A, Mayo J, Martinez E. Pulmonary gangrene secondary to pneumococcal pneumonia in a patient with AIDS. Clin Infect Dis 1997;24:268-9.

11. Schamaun M, von Buren U, Pirozynski W. [Massive lung necrosis in klebsiella pneumonia (so-called massive lung gangrene)]. Schweiz Med Wochenschr 1980;110:223-5.

12. Yangco BG, Deresinski SC. Necrotizing or cavitating pneumonia due to Streptococcus Pneumoniae: Report of four cases and review of the literature. Medicine (Baltimore) 1980;59:449-57.
13. Lopez-Contreras J, Ris J, Domingo P, Puig M, Martinez E. Tuberculous pulmonary gangrene: Report of a case and review. Clin Infect Dis 1994;18:243-5.

14. Khan FA, Rehman M, Marcus P, Azueta V. Pulmonary gangrene occurring as a complication of pulmonary tuberculosis. Chest 1980;77:76-80.

15. Donnelly L, Klosterman L. The yield of CT of children who have complicated pneumonia and noncontributory chest radiography. AJR Am J Roentgenol 1998;170:1627-31.

16. Reich JM. Pulmonary gangrene and the air crescent sign. Thorax 1993;48:70-4.

17. Delarue NC, Pearson FG, Nelems JM, Cooper JD. Lung abscess: Surgical implications. Can J Surg 1980;23:297-302.

18. Hoffer FA, Bloom DA, Colin AA, Fishman SJ. Lung abscess versus necrotizing pneumonia: Implications for interventional therapy. Pediatr Radiol 1999;29:87-91.

19. Hsu HS, Chern MS, Chen CH, Perng RP. Pulmonary gangrene: A case report. Zhonghua Yi Xue Za Zhi (Taipei) 1995;55:476-80.

20. Phillips LG, Rao KV. Gangrene of the lung. J Thorac Cardiovasc Surg 1989;97:114-8.

21. Lesnitskii LS, Kostiuchenko AL, Tulupov AN. [Several problems of pathogenesis and treatment of pulmonary gangrene]. Grudn Khir 1989:39-44.

22. Odell JA, Buckels NJ. Techniques of pneumonectomy. Pneumonectomy through an empyema. Chest Surg Clin N Am 1999;9:369-78,x-xi.

23. Refaely Y, Weissberg D. Gangrene of the lung: Treatment in two stages. Ann Thorac Surg 1997;64:970-4.

24. Sancho LM, Paschoalini MS, Fernandez A, Higutchi C, Jatene FB. [Surgical treatment of lung abscesses]. Rev Hosp Clin Fac Med Sao Paulo 1997;52:254-7. 


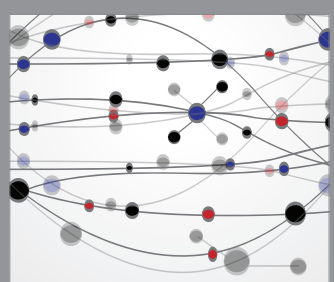

The Scientific World Journal
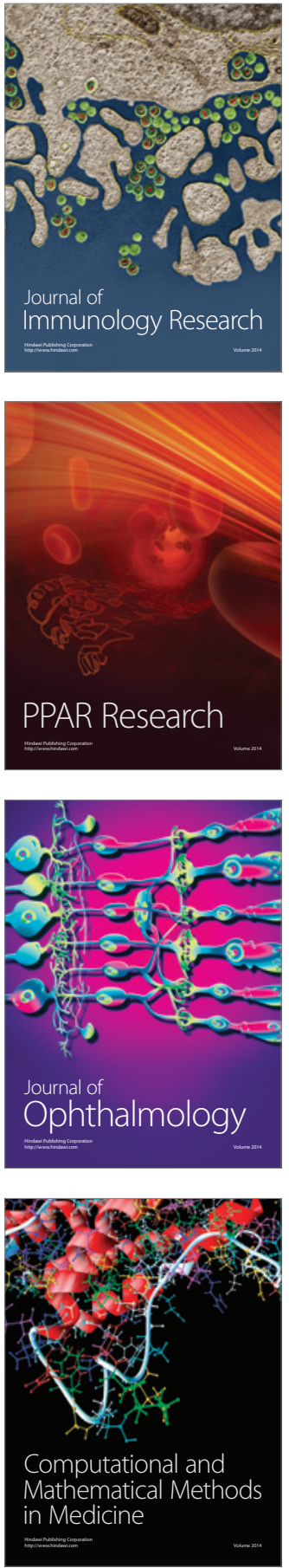

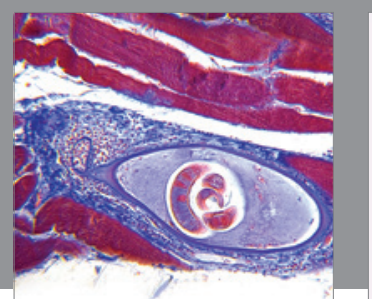

Gastroenterology Research and Practice

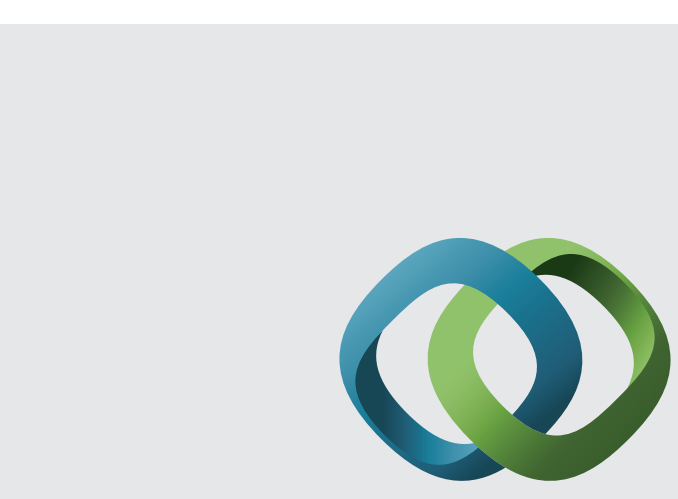

\section{Hindawi}

Submit your manuscripts at

http://www.hindawi.com
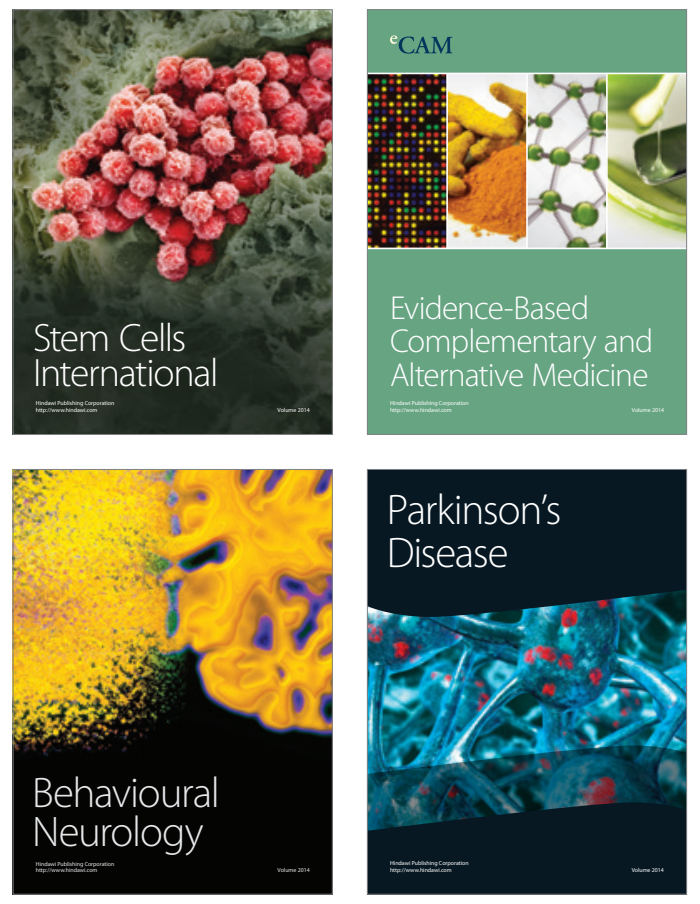
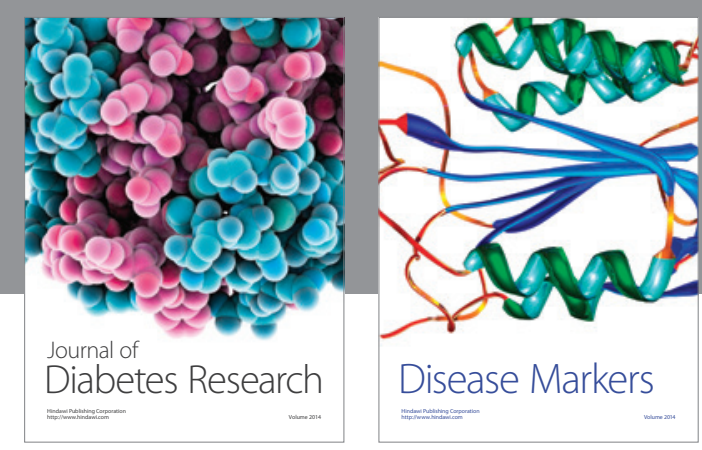

Disease Markers
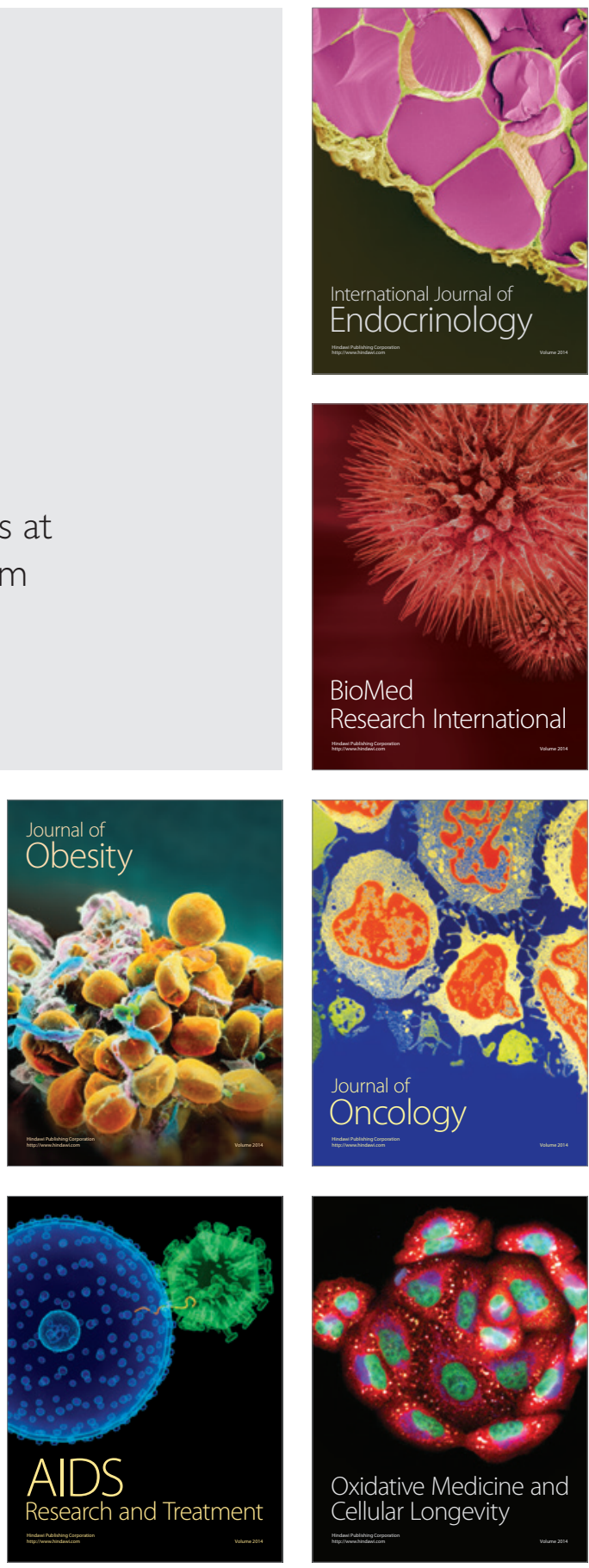